\title{
Shockwave Detection for Electronic Vehicle Detectors
}

\author{
Hsung-Jung Cho* and Ming-Te Tseng \\ Department of Transportation Technology and Management, \\ National Chiao Tung University, \\ Taiwan \\ hjcho@cc.nctu.edu.tw
}

\begin{abstract}
Although shockwaves have been extensively adopted in traditional traffic flow theory, how to detect shockwaves using an electronic vehicle detector has not been explored. Therefore, this study illustrates, for the first time, not only how to detect shockwaves, but also how to obtain shockwaves from three new traffic parameters: Stopped, Moving, and Empty. The Stopped parameter attempts to identify a newly arrived shockwave equation when a traffic queue approaches the electronic vehicle detector. The Moving and Empty parameters derive another new arrival shockwave equation when the electronic vehicle detector fails to identify any queue. An algorithm is also created to demonstrate how to use these parameters and equations to detect shockwaves. Additionally, numerous simulations are conducted to identify the behaviors of new traffic parameters and the effectiveness of the proposed algorithm. Results of this study demonstrate that the computing algorithm for electronic vehicle detectors can accurately detect shockwaves.
\end{abstract}

Keywords: Shockwave, electronic vehicle detector, stopped, moving, empty.

\section{Introduction}

Shockwave analysis has long been adopted in traffic flow analysis.[1] Shockwaves are defined as boundary conditions in the time-space domain that indicate a discontinuity in flow-density conditions.[2] Shockwaves result from sudden temporal or spatial changes in roadway density due to capacity or volume changes. Shockwave analysis is an effective means of analyzing flow and queuing problems.[3,4] Numerous techniques have been proposed via which shockwaves can be plotted and employed to forecast traffic system performance.[1] Applications to freeway bottlenecks and to traffic signals have also been developed.[5,6,7]

Most previous analyses and applications obtain shockwave values either via direct provision or by computation based on changes in flow and density (shockwave, $w=\Delta q / \Delta k)$. No previous investigation has studied methods for direct shockwave detection for electronic vehicle detectors. This investigation is the first to illustrate a method for shockwave detection for electronic vehicle detectors. Moreover, this investigation obtains, for the first time, shockwaves from three new traffic parameters

* Corresponding author.

Y. Shi et al. (Eds.): ICCS 2007, Part IV, LNCS 4490, pp. $275-282,2007$.

(C) Springer-Verlag Berlin Heidelberg 2007 
besides flow and density. The three new traffic parameters are Stopped, Moving, and Empty. Two shockwave equations are derived from these three parameters. When a traffic queue reaches an electronic vehicle detector, the shockwave equation can be derived from the Stopped parameter. When no traffic queue is present at an electronic vehicle detector, the Moving and Empty parameters are used to obtain another shockwave equation. An algorithm is also devised to demonstrate how to use these parameters and equations. Finally, CORSIM simulations were run for different combinations of scenarios to identify the behavior of new traffic parameters and the effectiveness of the computing algorithm.

\section{Formulation}

The formulation comprises four components. The first is the definitions of three new traffic parameters: Stopped, Moving and Empty. The second is the use of Stopped to detect shockwaves. The third is the use of Moving and Empty to detect shockwaves. The fourth is the algorithm, which combines the three new traffic parameters and two shockwave equations for shockwave detection.

\subsection{Three Traffic Parameters: Stopped, Moving and Empty}

Figure 1 provides the definitions of Stopped, Moving and Empty. Stopped refers to the detection of a vehicle that continues beyond a specified time interval. Moving means that the presence of a vehicle continues for less than a specified time interval. Empty means that no presence of a vehicle is detected during a specified time interval. If the specified time interval is short and the traffic flow is high, the short Moving and short Empty states alternate. Figure 1 shows the high sensitivity electronic detector. If the specified time interval is long and the traffic flow is high, the situation alternates between the long Stop, long Moving, and short Empty states. Figure 1 also illustrates the low sensitivity electronic detector. An electronic detector can have a low sensitivity, high sensitivity or both.

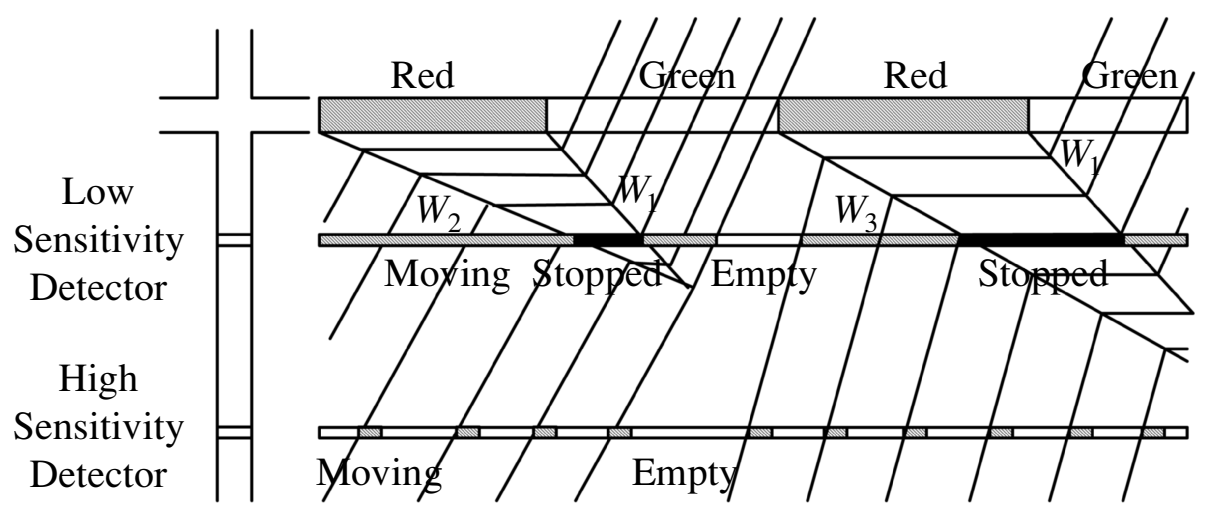

Fig. 1. Definitions of Stopped, Moving and Empty at a signalized intersection 


\subsection{Using Moving and Empty Time for Shockwave Detection}

When a high sensitivity electronic detector receives only Moving and Empty time, the traffic queue does not reach the electronic detector. Figure 2 illustrates the Moving time, vehicle and detection zones for such a traffic condition. Two vehicles with speed $\mathrm{V}$ and length $L_{V}$ pass through a high sensitivity electronic detector with zone length $L_{Z}$. Each vehicle triggers the moving time as $\left(L_{v}+L_{z}\right) / v$. Intuitively, the summary of the Moving time is related to traffic flow. Hence, the arrival shockwave $W_{3}$ during time interval $\Delta T$ can be expressed as

$$
W_{3}=\frac{L_{\text {Queue }}}{\Delta T}=\left(\sum_{n} \frac{L_{v}+L_{z}}{v}\right) \frac{C}{\text { Moving }+ \text { Empty }}=C \frac{\text { Moving }}{\text { Moving }+ \text { Empty }} .
$$

where $\mathrm{C}$ is a constant given by

$$
C=\frac{v\left(A v g L_{V}+A v g L_{g a p}\right)}{A v g L_{V}+L_{Z}} .
$$

where $L_{\text {Queue }}$ denotes the queue length for arriving vehicles, $A v g L_{V}$ represents the average vehicle length and $A v g L_{g a p}$ is the average gap length between two stopped vehicles.
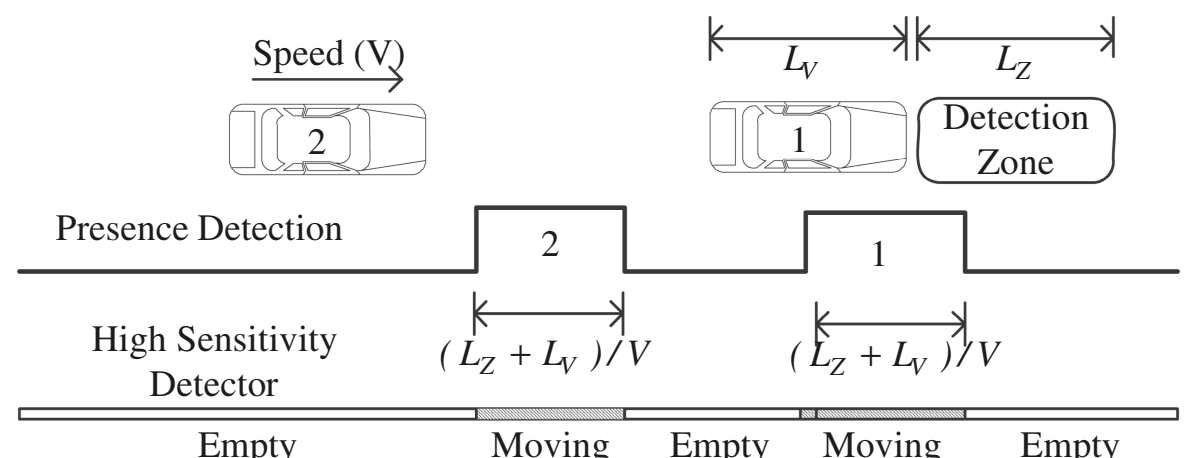

Fig. 2. Moving time, vehicles and detection zone

\subsection{Using Stopped Time for Shockwave Detection}

When traffic flow increases, traffic queue length gradually increases. If the traffic queue extends back to the position of the electronic vehicle detector during a red phase of a signalized intersection, the Stopped time is triggered. Generally, the Stopped time of the electronic detector changes slowly for continued signal cycles. Figure 3 displays the Stopped time for two continued signal cycles. The Stopped time of the first cycle is $\overline{O A}$, while that of the second cycle is $\overline{O C}$. The Stopped difference 


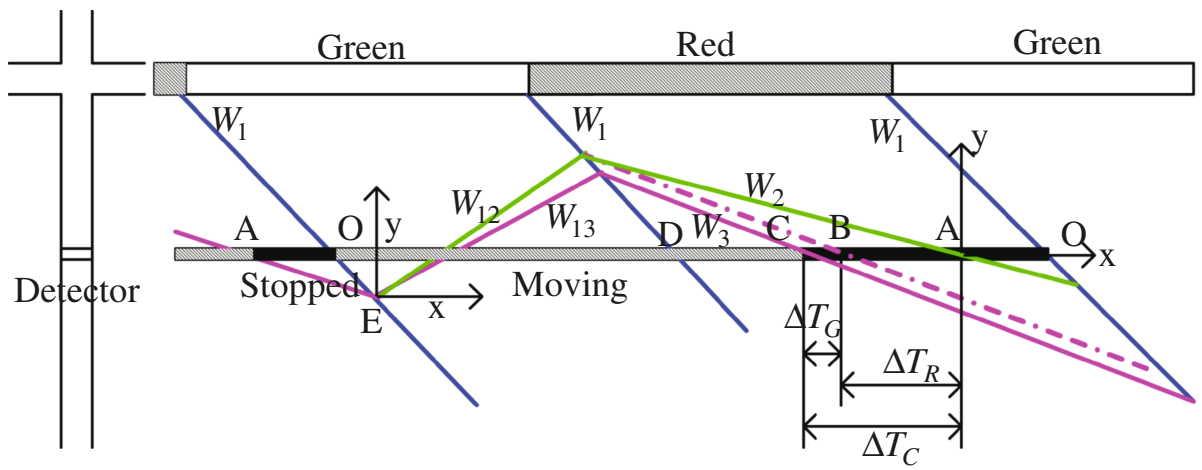

Fig. 3. Arrival shockwave and Stopped time

for these two continued cycles is $\overline{A C}$, or $\Delta T_{C}$. Meanwhile, $\overline{A C}$ is the sum of $\overline{A B}$ and $\overline{B C}$. Furthermore, $\overline{B C}$, or $\Delta T_{G}$, results from the difference in flow between shockwave $W_{12}$ and shockwave $W_{13}$ during the green phase $G$. Moreover, $\overline{A B}$, or $\Delta T_{R}$, results from the flow difference between shockwave $W_{2}$ and shockwave $W_{3}$ during the red phase $R$. Furthermore, $W_{3}$ denotes the shockwave of an arrival flow, while $W_{2}$ represents the shockwave of a special arrival flow in which total queued vehicles during the red phase can be discharged based only on the green phase time (in Fig.1). $W_{1}$ is the shockwave of the saturated discharge flow.

Therefore

$$
\Delta T_{C}=\Delta T_{G}+\Delta T_{R}
$$

Let point $\mathrm{A}$ be $(0,0)$ and set the $\mathrm{x}-\mathrm{y}$ coordinate axis as in Fig.3. By using linear algebra,

$$
\Delta T_{R}=\frac{W_{1} R\left(W_{3}-W_{2}\right)}{W_{3}\left(W_{1}-W_{2}\right)} .
$$

Similarly, let point $\mathrm{E}$ be $(0,0)$ and set the $\mathrm{x}-\mathrm{y}$ coordinate axis. Thus,

$$
\Delta T_{G}=\frac{W_{1} G\left(W_{1}-W_{3}\right)\left(W_{12}-W_{13}\right)}{W_{3}\left(W_{1}+W_{12}\right)\left(W_{1}+W_{13}\right)} .
$$

Suppose the traffic flow follows the model of Greenshield [8]:

$$
U=U_{f}\left(1-\frac{K}{K_{j}}\right) .
$$

where $U$ denotes speed, $U_{f}$ represents free flow speed, $K$ is density, and $K_{j}$ denotes traffic jam density. The following equations are then obtained: 


$$
W_{12}=\frac{R W_{1} W_{2}}{G W_{1}-C W_{2}} ; W_{12}=U_{f}-W_{1}-W_{2} ; W_{13}=U_{f}-W_{1}-W_{3} .
$$

Substituting $W_{12}$ and $W_{13}$ (Eq.7) into Eq.(5) yields the new $\Delta T_{G}$ :

$$
\Delta T_{G}=\frac{\left(G W_{1}-C W_{2}\right)^{2}\left(W_{3}-W_{1}\right)\left(W_{3}-W_{2}\right)}{\left(W_{1}-W_{2}\right) W_{3}\left(\left(G W_{1}-C W_{2}\right) W_{3}+C W_{2}^{2}-G W_{1}^{2}\right)} .
$$

To summarize, the following equation can be obtained.

$$
\Delta T_{C}=\frac{\left(G W_{1}-C W_{2}\right)^{2}\left(W_{3}-W_{1}\right)\left(W_{3}-W_{2}\right)}{\left(W_{1}-W_{2}\right) W_{3}\left(\left(G W_{1}-C W_{2}\right) W_{3}+C W_{2}^{2}-G W_{1}^{2}\right)}+\frac{W_{1} R\left(W_{3}-W_{2}\right)}{W_{3}\left(W_{1}-W_{2}\right)}
$$

where parameters $\Delta T_{C}, G, R, W_{1}$, and $W_{2}$ are all constants. Thus it is easy to obtain $W_{3}=f\left(\Delta T_{C}, G, R, W_{1}, W_{2}\right)$.

\subsection{Algorithm}

The previous subsections propose two arrival shockwave equations. Equation (1) demonstrates how to use Moving and Empty time to detect arrival shockwave.

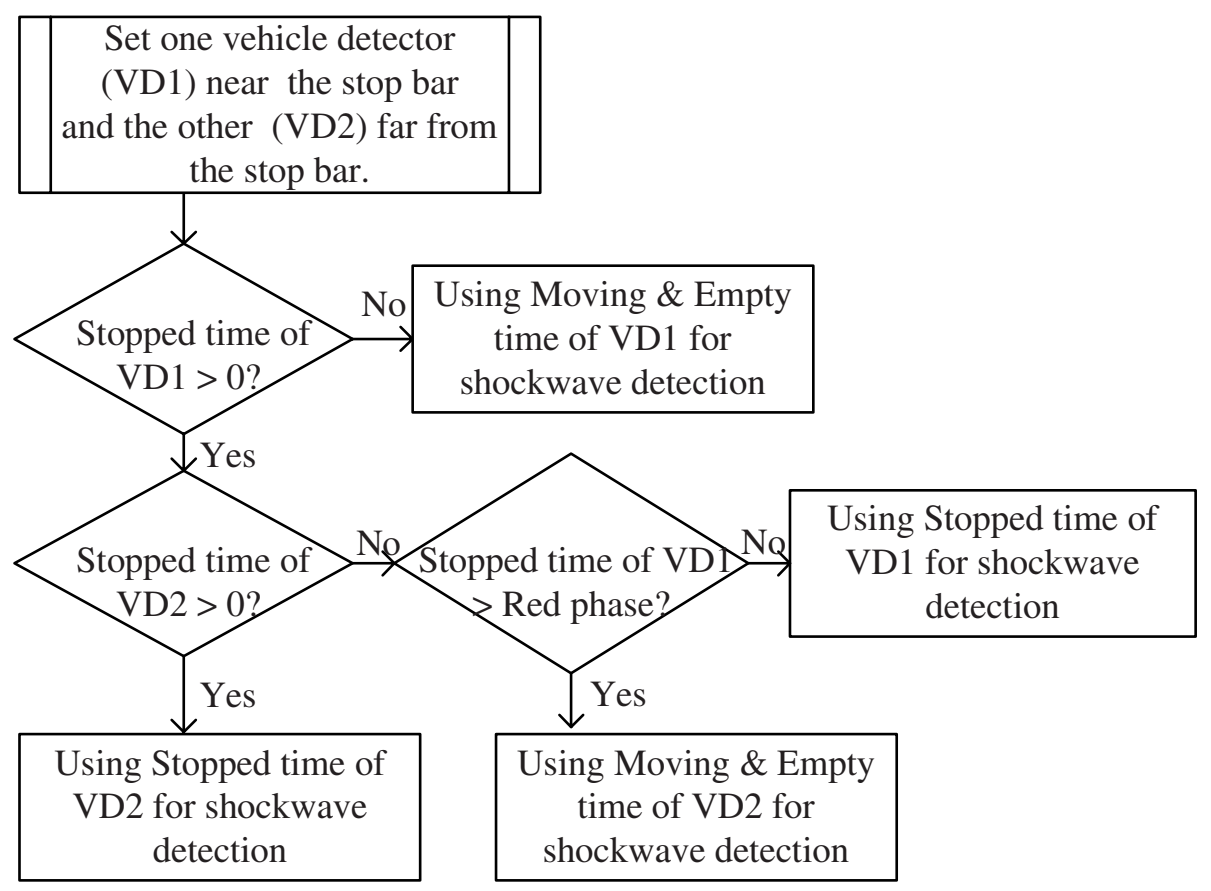

Fig. 4. Shockwave detection algorithm 
Meanwhile, Equation (9) demonstrates how to use Stopped time to detect arrival shockwave. In Fig.4, the algorithm illustrated how to combine these two equations for arrival shockwave detection.

\section{Simulation Results}

Two CORSIM frameworks have been created for assessing the three new traffic parameters and the proposed shockwave detection algorithm. One framework is designed to identify the relations between environment and the three new traffic parameters. The other is for testing the shockwave detection algorithm.

\subsection{Three Traffic Parameters and Environment}

The test example shown in Table 1 involves two adjacent intersections with a 700 feet link at a fixed-timed traffic signal operating with a 60-s cycle length and a 23 -s effective green interval. To determine the features of the three parameters and the effects of environment change, eight scenarios were prepared and listed in Table 1: traffic flow changing from $48 \mathrm{vph}$ to $2880 \mathrm{vph}$, different detector zone size (20, 50 feet), different vehicle detector distances to the stop bar (0,30, 60,90,120,240 feet), and next intersection spill back condition. Table 1 lists the test results. The arrows in Table 1 indicate the trend of traffic parameters.

Table 1. Relation between three traffic parameters and the environment

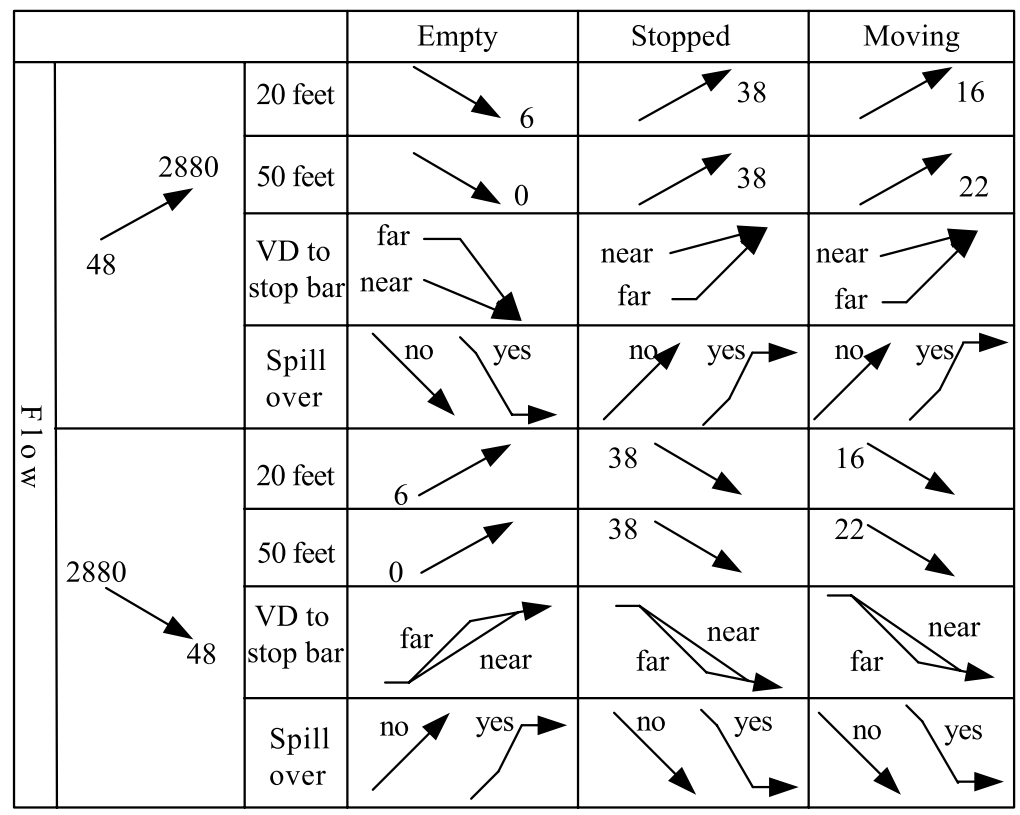




\subsection{Shockwave Detection}

The other test example for arrival shockwave features two adjacent intersections with a 997 foot link at a fixed-timed traffic signal operating with a 60-s cycle length and a 30 -s effective green interval. Two electronic vehicle detectors are located 300 and 570 feet from the stop bar. The change sequence for the traffic flow is 650,550,600, $500,700,550,400,600$ and $1000 \mathrm{vph}$ for every 900 seconds. Since the capacity is 600 $\mathrm{vph}$, the v/c values vibrate around 1 and some traffic queues are formed. Figure 5 shows three traffic parameters in two electronic vehicle detectors. Notably, the Stopped time of VD2 is always zero, indicating that no traffic queue exists over VD2. Furthermore, the Stopped time of VD1 is occasionally near the effective red time (30). Restated, some traffic queues are shown on VD1. Figure 6 summarizes the results from two different shockwave equations proposed in subsections 2.2 and 2.3. According to this figure, each equation can only detect arrival shockwaves accurately during a specific time interval. Figure 7 illustrates that the algorithm can obtain good results. The detected arrival shockwave is almost identical to Corsim's shockwave.

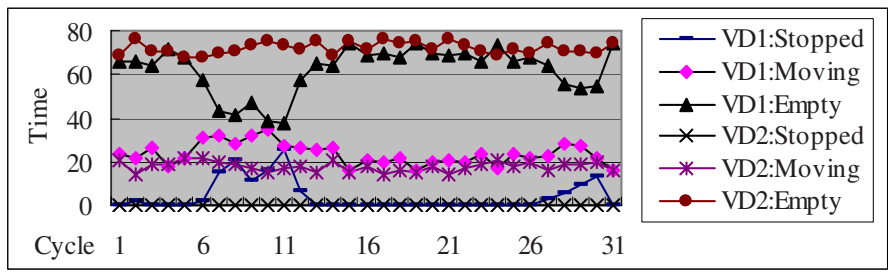

Fig. 5. Three traffic parameters in VD1 and VD2

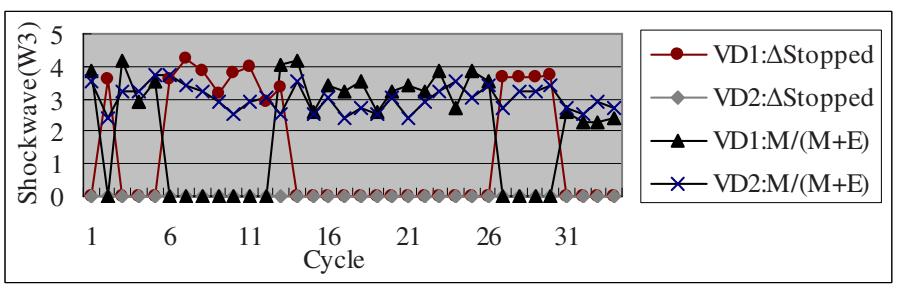

Fig. 6. Using Stopped, Moving and Empty of VD to detect arrival shockwave

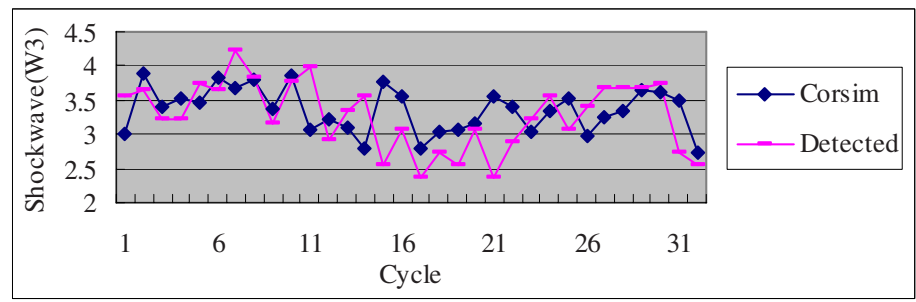

Fig. 7. The final result of the shockwave detection algorithm 


\section{Conclusions}

This work proposes, for the first time, three new traffic parameters and a new approach to shockwave detection for electronic vehicle detectors. The proposed shockwave detection approach comprises three major components: a shockwave equation derived from the new Stopped parameter, the other shockwave equation derived from the new Moving and Empty parameters, and an algorithm showing how to use the new parameters and equations to more accurately identify shockwaves. Different scenarios involving simulated environments were used to identify the behaviors of the three traffic parameters. The proposed shockwave detection methodology was tested for a case involving two adjacent intersections in a simulated environment. Simulated data from two electronic vehicle detectors were used. Each electronic vehicle detector obtained two Shockwave equations. The proposed algorithm selected the best of two equations as the final shockwave. This study has demonstrated the feasibility of using the two shockwave equations and algorithm for shockwave detection in urban intersections. The proposed algorithm is easily applied and cost-effective since low cost electronic detectors can be used to generate these three new parameters and detect shockwaves. Further research is required to extend the concept to freeways and highways.

\section{Acknowledgements}

The authors would like to thank the Ministry of Education of the Republic of China, Taiwan (MOE ATU Program) and the National Science Council of the Republic of China, Taiwan (Contract No. NSC-95-2221-E-009-346, NSC-95-2221-E-009-347 and NSC-95-2752-E-009-010-PAE) for financially supporting this research.

\section{References}

1. Gazis, D.C.:The Origins of Traffic Theory, Operation Research, Vol. 50, No. 1, 2002, pp. 69-77.

2. May, A. D.:Traffic Flow Fundamentals, Prentice Hall, Englewood Cliffs, New Jersey 07632, 1990.

3. Zhang, H.M.: A theory of nonequilibrium traffic flow, Transportation Research B 32 (7), 1998, pp. 485-498.

4. Cho, H.-J., Lo, S. C.: Modeling of Self-consistent Multi-class Dynamic Traffic Flow Model, Physica A., Vol. 312, 2002, pp. 342-362.

5. Cho, H.-J., Tseng, M.-T.: A Novel Computational Algorithm for Traffic Control SoC, WSEAS Transactions on Mathematics, Issue 1, Volume 5, 2006, pp. 123-128.

6. Dion, F., Rakha, H., Kang, Y.-S.: Comparison of delay estimates at under-saturated and over-saturated pre-timed signalized intersections, Transportation Research-B, Volume 38, 2004, pp. 99-122.

7. Abu-Lebdeh, G., Benekohal, R. F.: Development of Traffic Control and Queue Management Procedures for Oversaturated Arterials, Transportation Research Record, No. 1603, 1997, pp. 119-127.

8. Greenshields, B.D.: A study of traffic capacity. Proceedings of the Highway Research Board 14, 1934, pp. 448-477. 